\title{
DETERMINAÇÃO DO TEOR DE LUTEÍNA EM HORTALIÇAS
}

\author{
ALINE MANKE NACHTIGALL* \\ PAULO CESAR STRINGHETA**

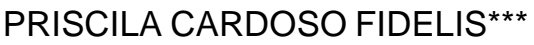 \\ FABIANE MANKE NACHTIGALL****
}

O objetivo do presente trabalho foi avaliar o teor de luteína em algumas hortaliças comumente consumidas no estado de Minas Gerais (BRASIL), visando fornecer dados para que a população possa conhecer as melhores fontes desse carotenóide. A extração do pigmento foi realizada com acetona, etanol, hexano e tetraidrofurano, por 24h. Após a extração realizou-se a saponificação com $\mathrm{KOH}$ etanólico $10 \%$ para eliminar impurezas, como clorofila e ácidos graxos. O teor de luteína nas hortaliças foi determinado por medidas espetrofotométricas e confirmado por cromatografia a líquido de alta eficiência (CLAE). Pelos resultados obtidos, a acetona foi o solvente mais efetivo para a extração do pigmento. O hexano não se mostrou eficiente para extração de vegetais frescos nessas condições. Couve, rúcula, agrião, mostarda, acelga, espinafre, azedinho e brócolis constituem fontes ricas em luteína. Deve-se ressaltar o elevado teor desse carotenóide no lobrobô (também conhecido como orapro-nobis) na serralha, no almeirão e na taioba, vegetais não-convencionais e de consumo regionalizado.

PALAVRAS-CHAVE: LUTEÍNA; CAROTENÓIDES; HORTALIÇAS.

* Química de Alimentos, Doutoranda em Ciência e Tecnologia de Alimentos, Universidade Federal de Viçosa (UFV), Viçosa, MG (e-mail: anachtigall@gmail.com).

** Professor, Doutor em Ciência de Alimentos, DTA, UFV, Viçosa, MG (e-mail: pstringheta@yahoo.com.br).

*** Aluna do Curso de Engenharia de Alimentos, UFV, Viçosa, MG (e-mail: priscila_cf@yahoo.com).

*** Química, Doutoranda em Química, Universidade Estadual de Campinas (UNICAMP), Campinas, SP (email: fabimanke@yahoo.com.br). 


\section{INTRODUÇÃO}

Dados do Conselho Brasileiro de Oftalmologia estimam que, aproximadamente, 2,9 milhões de brasileiros com mais de 65 anos de idade apresentam casos de degeneração macular relacionada à idade (DMRI). Essa doença ocular, grave e irreversível, causa cegueira em pessoas na faixa etária acima de 65 anos e com o aumento da expectativa de vida é natural que o número de casos se eleve (LAJOLO, 2004).

A luteína constitui potente antioxidante que previne danos causados pelos radicais livres nos tecidos (ALVES-RODRIGUES e SHAO, 2004; SILVA, 2004). Esse carotenóide absorve a luz azul prejudicial aos olhos e com isso reduz em $40 \%$ a incidência de luz danosa à mácula, região localizada no centro da retina, responsável pela visão nítida das imagens (DAGNELIE, ZORGE e MCDOANLD, 2000). Pessoas com mais de 60 anos e reduzida acuidade visual apresentam baixos níveis de luteína e zeaxantina na região macular, os dois carotenóides majoritários presentes no olho (YEUM et al., 1995). Em contraste, pessoas com a mesma idade e elevada densidade de pigmentos maculares apresentam sensibilidade visual similar à de jovens. A perda da sensibilidade visual pode ser o precursor de algumas doenças dos olhos, incluindo a DMRI.

Dentre os principais benefícios associados à luteína, além de prevenir a DMRI, destacamse a prevenção da aterosclerose, da catarata, do câncer de cólon e de outras patologias (ALVESRODRIGUES e SHAO, 2004; BROW et al., 1999; CHASAN-TABER et al., 1999; DAGNELIE, ZORGE e McDOANLD, 2000; DELI et al., 2004; SUMANTRAN et al., 2000).

Os seres humanos não apresentam a capacidade de sintetizar carotenóides, sendo necessária dieta balanceada incluindo o consumo de alimentos ricos nesses compostos. Estudos realizados com animais e humanos demonstram que a concentração de luteína no sangue e nos tecidos está estreitamente relacionada ao consumo de alimentos ricos desse carotenóide, tais como frutas e hortaliças de folhas verdes (MERCADANTE e RODRIGUEZ-AMAYA, 2001; SILVA, 2004; STRUNCH, 2004). Todavia, deve-se levar em consideração que tais fontes vegetais contêm carotenóides específicos de acordo com sua espécie.

A quantidade total e a concentração relativa de cada carotenóide podem ser influenciadas pela seleção de cultivares e variedades. Por essa razão, é necessário cautela no uso de dados de trabalhos e de tabelas de composição de alimentos. Essas informações dependem de outros fatores como parte da planta consumida, estágio de maturação, condições climáticas e geográficas e manejo pós-colheita (KIMURA e RODRIGUEZ-AMAYA, 1993; RODRIGUEZ-AMAYA, 2001; SÁ e RODRIGUEZ-AMAYA, 2003).

O objetivo deste trabalho foi explorar novas fontes de luteína, bem como avaliar e comparar o conteúdo de fontes conhecidas com dados existentes na literatura. Com o levantamento dessas informações será possível auxiliar a decisão de compra dos consumidores de forma a incrementar a dieta com alimentos ricos em luteína.

\section{MATERIAL E MÉTODOS}

\subsection{AMOSTRAS}

As seguintes hortaliças foram compradas no comércio de Viçosa/MG: acelga (Beta vulgaris), agrião (Nasturtium officinale), almeirão (Cichorium intybus), azedinho (Taraxacum officinale Webber ex F.H. Wigg), brócolis (Brassica oleracea var itálica), couve (Brassica oleracea car acephala), couve-roxa (Brassica oleracea var acephala), espinafre (Tetragonia expansa), jiló (Solanum gilo), lobrobô ou orapro-nobis (Pereskia aculeata), mostarda (Brassica juncae), pimentão verde, vermelho e amarelo (Capsicum Annuum), quiabo (Hibiscus esculentis), repolho (Brassica oleracea var capitata), rúcula (Eruca sativa), serralha (Sonchus oleraceus), taioba (Xanthosoma sagittifolium) e vagem (Phaseolus vulgaris). 


\subsection{EXTRAÇÃO DOS PIGMENTOS}

O método para extração dos carotenóides totais envolveu a combinação dos procedimentos descritos por KIMURA e RODRIGUEZ-AMAYA (2002) e PEREIRA (2002).

No processo de extração empregaram-se os seguintes solventes: acetona, etanol, hexano e tetraidrofurano. Todos os procedimentos foram realizados em ambiente escuro de acordo com a Figura 1.

FIGURA 1 - FLUXOGRAMA DE EXTRAÇÃO DE CAROTENÓIDES

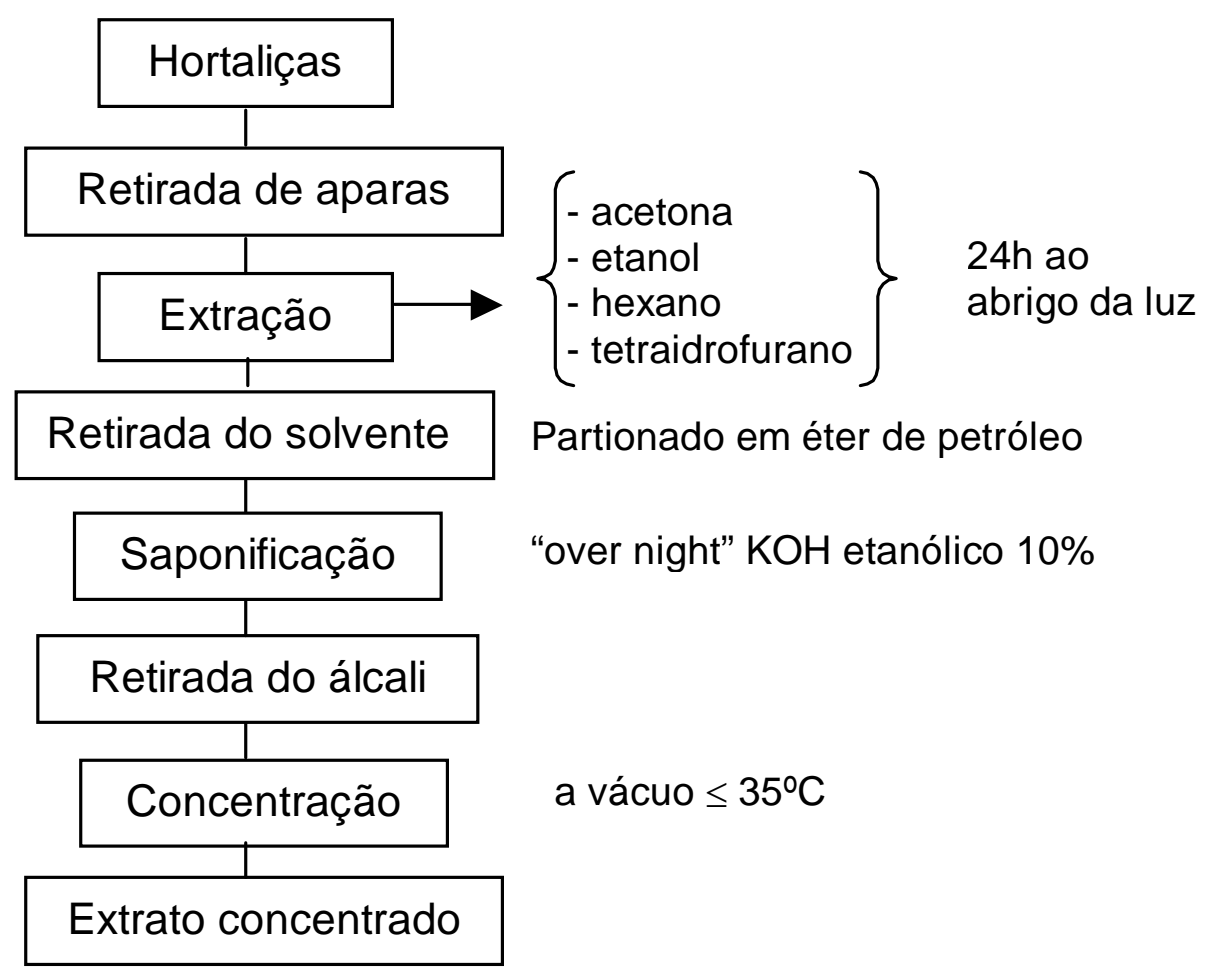

Imediatamente após a aquisição, as hortaliças foram levadas ao laboratório. Procedeu-se a separação das partes comestíveis, que foram cortadas finamente com faca inoxidável. Retirou-se uma amostra de aproximadamente 5,0 gramas, peso exato de cada material, aos quais foram acrescentados $50 \mathrm{~mL}$ de cada solvente. Após $24 \mathrm{~h}$ de extração ao abrigo da luz, as amostras foram filtradas em funil de Büchner a vácuo, repetindo-se o procedimento até todo o resíduo tornar-se incolor. Em seguida, os pigmentos foram transferidos em pequenas porções para éter de petróleo em funil de separação para a retirada do solvente. Efetuou-se a saponificação com $\mathrm{KOH}$ etanólico $10 \%$ "over night". Após a retirada do álcali concentrou-se o extrato de carotenóides a vácuo em temperatura de $\leq 35^{\circ} \mathrm{C}$, finalizando a evaporação com fluxo de $\mathrm{N}_{2}$. Todos os procedimentos foram realizados em ambiente escuro.

\subsection{QUANTIFICAÇÃO DE CAROTENÓIDES TOTAIS}

Efetuou-se a quantificação do teor de carotenóides totais nas diferentes amostras por medidas espectrofotométricas, utilizando o coeficiente de extinção $E_{1 \mathrm{~cm}}^{1 \%}=2500$ em etanol com leitura no $\lambda$ máx. As análises foram realizadas em espectrofotômetro ultra-violeta/visível (UV/VIS) digital, marca HITACHI, modelo U-2001, seguindo metodologia descrita por CISNEROS et al. (2004). 


\subsection{CONFIRMAÇÃO DA IDENTIDADE DOS CAROTENÓIDES}

A confirmação qualitativa da presença de luteína nas amostras foi efetuada por cromatofrafia a líquido de alta eficiência (NUNES e MERCADANTE, 2004), empregando cromatógrafo a líquido Shimadzu CLASS-VP. Os carotenóides foram separados em coluna de fase reversa $\mathrm{C}_{30}$ YMC ( $5 \mu \mathrm{m}, 250 \mathrm{~mm} \times 4,6 \mathrm{~mm}$ ), utilizando metanol (0,1\% de trietilamina)/ metil-t-butil éter 95:5 como fase móvel e fluxo de $1 \mathrm{~mL}$. $\mathrm{min}^{-1}$ em modo isocrático, injeção de $30 \mu \mathrm{L}$ e temperatura da coluna variando entre 29 e $33^{\circ} \mathrm{C}$. Os cromatogramas foram obtidos a $450 \mathrm{~nm}$.

Baseou-se a caracterização dos carotenóides na ordem de eluição na coluna $C_{30}$ pela comparação das características espectrofotométricas no UV-VIS com dados da literatura e com padrão de luteína.

Os solventes utilizados, todos grau cromatográfico, foram previamente filtrados em sistema Millipore de filtração a vácuo com membrana de 0,45 $\mu \mathrm{m}$ (apropriada para solvente orgânico) e degaseificados em ultra-som. As amostras foram filtradas em membrana de polietileno de 0,22 $\mu \mathrm{m}$ de poro.

Calculou-se a concentração de luteína nas amostras vegetais a partir dos dados cromatográficos, sendo o teor de luteína estimado pela percentagem de luteína em relação ao total de carotenóides.

\subsection{DELINEAMENTO EXPERIMENTAL}

Adotou-se delineamento inteiramente casualizado num total de 80 tratamentos, provenientes de fatorial de 20 vegetais e 4 solventes extratores $(20 \times 4)$, com três repetições.

Para verificar a existência de diferenças significativas entre os tratamentos realizou-se a análise de variância, seguida pelo teste de Duncan $(p \leq 0,05)$ para avaliar as diferenças entre as médias. As análises foram efetuadas com o auxílio do Software SANEST (ZONTA e MACHADO, 1991).

\section{RESULTADOS E DISCUSSÃO}

Para avaliar a capacidade extratora dos solventes e a potencialidade de cada material vegetal realizou-se a análise estatística para os solventes com relação à espécie e para a espécie em relação ao solvente. Os teores de carotenóides totais encontram-se na Tabela 1.

Ao analisar a Tabela 1 percebe-se que a acetona apresentou maior poder extrator para a maioria das amostras estudadas. Esperava-se que o tetraidrofurano extraísse teor superior de luteína por apresentar poder de solubilização 10 vezes maior que o da acetona, 27 vezes o do etanol e 400 vezes o do hexano (CRAFT, 1992). No entanto, em conseqüência da maior polaridade do tetraidrofurano podem ter ocorrido muitas perdas do carotenóide durante as sucessivas lavagens na etapa de saponificação (SÁ e RODRIGUEZ-AMAYA, 2003).

A acetona apresentou poder extrator semelhante ao etanol para o almeirão e a serralha, similar ao do tetraidrofurano para azedinho e, análogo ao etanol e ao tetraidrofurano para agrião, acelga, lobrobô e taioba. Para as demais fontes avaliadas (couve comum, espinafre, mostarda e rúcula), a acetona mostrou-se o solvente mais efetivo. No entanto, os quatro solventes estudados apresentaram capacidade extratora similar para jiló, pimentão vermelho, pimentão verde, pimentão amarelo, quiabo, repolho e vagem.

Notou-se que o solvente com pior capacidade extratora foi o hexano. Tal fato pode ser justificado pela baixa solubilidade da luteína em hexano (CRAFT, 1992) e elevada quantidade de água nas hortaliças frescas, o que pode ter dificultado o contato do solvente com o pigmento a ser extraído. Desta forma, o hexano não se mostrou um solvente adequado para extração de carotenóides em hortaliças. 
TABELA 1 - TEOR DE CAROTENÓIDES TOTAIS (mg.100 $\mathrm{g}^{-1}$ DE PRODUTO FRESCO) NAS HORTALIÇAS DETERMINADO POR ESPECTROFOTOMETRIA $(E=\mathbf{2 5 0 0})$

\begin{tabular}{lllll}
\hline Produto & Acetona & \multicolumn{1}{c}{ Etanol } & Hexano & Tetraidrofurano \\
& & & & \\
\hline Acelga & $4,15 \mathrm{Abcd}$ & $3,45 \mathrm{Acde}$ & $0,51 \mathrm{Ba}$ & $2,72 \mathrm{Ade}$ \\
Agrião & $5,27 \mathrm{Ab}$ & $5,56 \mathrm{Aab}$ & $1,15 \mathrm{Ba}$ & $4,64 \mathrm{Aab}$ \\
Almeirão & $3,56 \mathrm{Acde}$ & $3,36 \mathrm{Acde}$ & $0,77 \mathrm{Ba}$ & $1,06 \mathrm{Bf}$ \\
Azedinho & $2,14 \mathrm{ABef}$ & $0,92 \mathrm{BCghi}$ & $0,09 \mathrm{Ca}$ & $3,06 \mathrm{Acde}$ \\
Brócolis & $1,31 \mathrm{Bfg}$ & $2,83 \mathrm{Aef}$ & $0,26 \mathrm{Ba}$ & $1,57 \mathrm{ABef}$ \\
Couve & $7,89 \mathrm{Aa}$ & $3,48 \mathrm{Bcde}$ & $1,28 \mathrm{Ca}$ & $3,99 \mathrm{Bbcd}$ \\
Couve roxa & $2,16 \mathrm{Bef}$ & $4,39 \mathrm{Abcd}$ & $0,04 \mathrm{Ca}$ & $0,65 \mathrm{Cf}$ \\
Espinafre & $3,53 \mathrm{Acde}$ & $1,41 \mathrm{Abcd}$ & $0,27 \mathrm{Ba}$ & $0,65 \mathrm{Bf}$ \\
Jiló & $0,10 \mathrm{Ag}$ & $0,06 \mathrm{Ai}$ & $0,01 \mathrm{Aa}$ & $0,08 \mathrm{Af}$ \\
Lobrobô & $4,83 \mathrm{Abc}$ & $6,07 \mathrm{Aa}$ & $0,46 \mathrm{Ba}$ & $5,77 \mathrm{Aa}$ \\
Mostarda & $4,30 \mathrm{Abcd}$ & $1,84 \mathrm{Bfgh}$ & $0,06 \mathrm{Ca}$ & $2,91 \mathrm{Bcde}$ \\
Pimentão & & & & \\
amarelo & $0,46 \mathrm{Ag}$ & $0,77 \mathrm{Aghi}$ & $0,34 \mathrm{Aa}$ & $0,38 \mathrm{Af}$ \\
Pimentão verde & $0,51 \mathrm{Ag}$ & $1,25 \mathrm{Aghi}$ & $0,45 \mathrm{Aa}$ & $0,61 \mathrm{Af}$ \\
Pimentão & & & & \\
vermelho & $0,77 \mathrm{Afg}$ & $1,24 \mathrm{Aghi}$ & $0,15 \mathrm{Aa}$ & $0,81 \mathrm{Af}$ \\
Quiabo & $0,89 \mathrm{Afg}$ & $1,06 \mathrm{Aghi}$ & $0,83 \mathrm{Aa}$ & $0,67 \mathrm{Af}$ \\
Repolho & $0,09 \mathrm{Ag}$ & $0,11 \mathrm{Ai}$ & $0,01 \mathrm{Aa}$ & $0,07 \mathrm{Af}$ \\
Rúcula & $7,18 \mathrm{Aa}$ & $2,21 \mathrm{Cefg}$ & $0,24 \mathrm{Da}$ & $4,31 \mathrm{Bbc}$ \\
Serralha & $5,44 \mathrm{Ab}$ & $4,92 \mathrm{ABabc}$ & $0,12 \mathrm{Ca}$ & $3,95 \mathrm{Bbcd}$ \\
Taioba & $3,17 \mathrm{Ade}$ & $3,45 \mathrm{Acde}$ & $0,63 \mathrm{Ba}$ & $2,59 \mathrm{Ade}$ \\
Vagem & $0,41 \mathrm{Ag}$ & $0,34 \mathrm{Ahi}$ & $0,15 \mathrm{Aa}$ & $0,20 \mathrm{Af}$ \\
& & & & \\
\hline
\end{tabular}

Médias seguidas por letras maiúsculas distintas nas linhas diferem entre si pelo Teste de Duncan, ao nível de 5\% de probabilidade, em relação aos diferentes solventes em função da espécie. Médias seguidas por letras minúsculas distintas nas colunas diferem entre si pelo Teste de Duncan, ao nível de $5 \%$ de probabilidade, em relação às diferentes espécies para determinado solvente.

Com relação à espécie em função do solvente extrator, o material vegetal que apresentou maior potencial em termos de carotenóides totais foi a couve comum. Essa hortaliça demonstrou elevada concentração de carotenóides ao ser extraída com acetona (7,89 mg.100 $\left.\mathrm{g}^{-1}\right)$ e menor teor ao ser extraída com os demais solventes (tetraidrofurano: 3,99 mg.100 g ${ }^{-1}$; etanol: 3,48 mg.100 g ${ }^{-1}$; hexano: $\left.1,28 \mathrm{mg} \cdot 100 \mathrm{~g}^{-1}\right)$.

Ao utilizar a acetona (Tabela 1 ), a couve comum $\left(7,89 \mathrm{mg} .100 \mathrm{~g}^{-1}\right)$ e a rúcula $\left(7,18 \mathrm{mg} .100 \mathrm{~g} \mathrm{~g}^{-1}\right)$ apresentaram os valores mais elevados de carotenóides. No entanto, para os solventes etanol e tetraidrofurano as fontes vegetais com as maiores concentrações de carotenóides foram o lobrobô $(6,07$ e 5,77 mg.100 g-1) e o agrião $(5,56$ e 4,64 mg. $\left.100 \mathrm{~g}^{-1}\right)$. Constata-se assim que os solventes apresentaram poder extrator diferenciado, influenciando a quantificação do teor de luteína nos vegetais.

Analisando o perfil cromatográfico dos extratos vegetais, conforme ilustrado pela Figura 2, verificou-se que a composição qualitativa permaneceu constante com variações quantitativas no perfil de carotenóides dos mesmos. 
FIGURA 2 - CROMATOGRAMAS DOS EXTRATOS DE (A) ALMEIRÃO, (B) LOBROBÔ, (C) SERRALHA E (D) TAIOBA, EXTRAÍDOS EM ACETONA
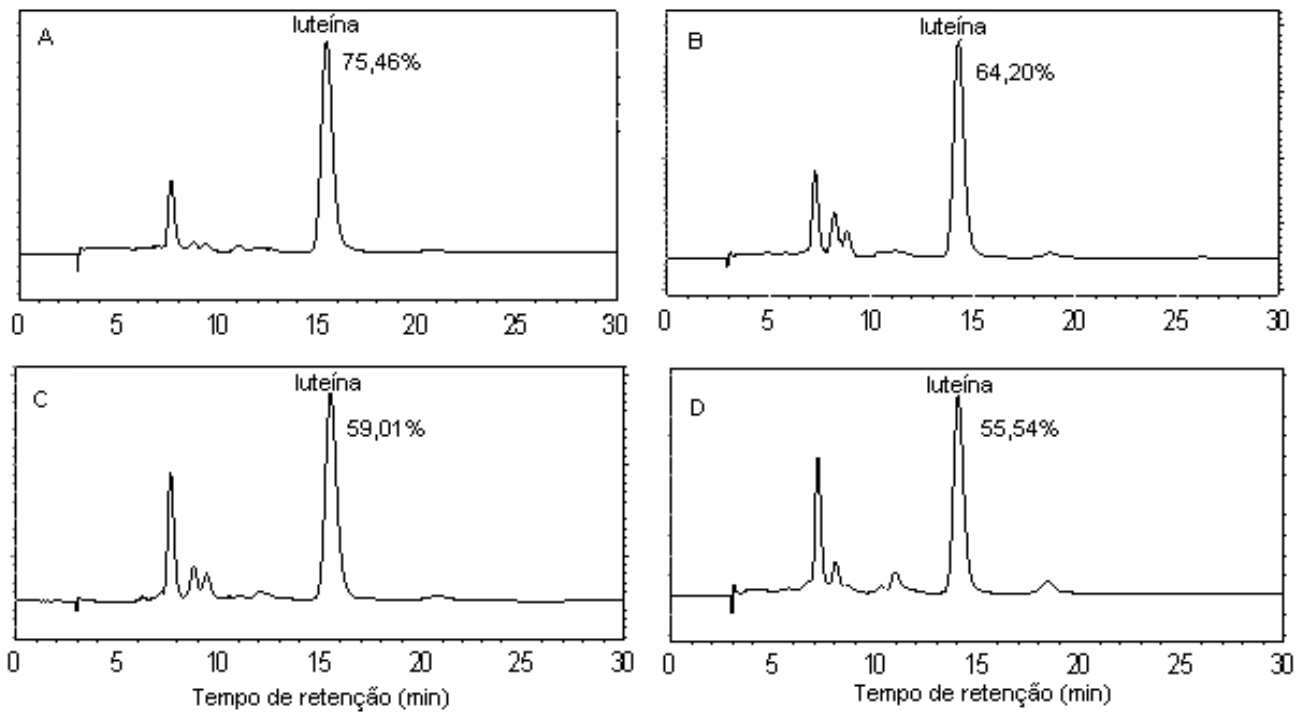

Coluna polimérica $\mathrm{C}_{30}$;

Fase móvel: metanol (0,1\% TEA)/ metil-t-butil éter;

Fluxo de $1 \mathrm{~mL} \cdot \mathrm{min}^{-1}$.

Ao quantificar extratos por espectrofotometria deve-se conhecer a identidade e proporção de cada componente dos mesmos para utilizar os valores específicos de $E$. Tendo em vista que as frações do extrato não foram identificadas em sua totalidade, até por questão de custo e tempo, o teor de luteína nas amostras foi estimado pela proporção de luteína em relação ao total de carotenóides obtida por CLAE (Tabela 2).

TABELA 2 - PORCENTAGEM (\%) DETERMINADA POR CLAE E CONCENTRAÇÃO DE LUTEÍNA (mg.100 g-1 DE PRODUTO FRESCO) NOS VEGETAIS

\begin{tabular}{lcccccccc}
\hline \multirow{2}{*}{ Produto } & \multicolumn{2}{c}{ Acetona } & \multicolumn{2}{c}{ Etanol } & \multicolumn{2}{c}{ Hexano } & \multicolumn{2}{c}{ Tetraidrofurano } \\
\cline { 2 - 8 } & $\%$ Lut. & Luteína & $\%$ Lut. & luteína & $\%$ Lut. & Luteína & $\%$ Lut. & luteína \\
\hline Acelga & 63,42 & 2,63 & 42,80 & 1,48 & 38,28 & 0,20 & 57,99 & 1,58 \\
Agriäo & 60,02 & 3,17 & 43,41 & 2,41 & 26,70 & 0,31 & 63,66 & 2,95 \\
Almeirẫo & 75,46 & 2,69 & 88,77 & 2,98 & 30,76 & 0,24 & 71,43 & 0,76 \\
Azedinho & 77,30 & 1,66 & 65,63 & 0,60 & 14,84 & 0,01 & 65,55 & 2,01 \\
Brócolis & 57,97 & 0,76 & 64,95 & 1,84 & 7,34 & 0,02 & 55,86 & 0,88 \\
Couve & 44,40 & 3,51 & 56,15 & 1,95 & 46,82 & 0,60 & 61,66 & 2,46 \\
Couve roxa & 68,60 & 1,48 & 83,02 & 3,64 & 46,65 & 0,02 & 62,90 & 0,41 \\
Espinafre & 62,55 & 2,21 & 48,21 & 0,68 & 6,39 & 0,02 & 65,21 & 0,43 \\
Jilóy & 86,72 & 0,09 & 89,71 & 0,05 & 80,99 & 0,01 & 14,84 & 0,01 \\
Lobrobô & 64,20 & 3,10 & 78,45 & 4,76 & 38,85 & 0,18 & 60,61 & 3,50 \\
Mostarda & 57,44 & 2,47 & 52,65 & 0,97 & 71,26 & 0,04 & 57,00 & 1,66 \\
Pimentẫo amarelo & 24,07 & 0,11 & 37,35 & 0,29 & 32,16 & 0,11 & 14,83 & 0,06 \\
Pimentấy verde & 76,23 & 0,39 & 68,40 & 0,86 & 31,05 & 0,14 & 70,10 & 0,43 \\
Pimentẫo vermelho & 54,66 & 0,42 & 61,09 & 0,76 & 47,43 & 0,07 & 48,98 & 0,39 \\
Quiabo & 28,96 & 0,26 & 68,99 & 0,73 & 19,90 & 0,16 & 58,25 & 0,39 \\
Repolho & 34,39 & 0,03 & 61,18 & 0,01 & 55,04 & 0,01 & 14,50 & 0,01 \\
Rúcula & 71,32 & 5,12 & 88,60 & 1,96 & 9,29 & 0,02 & 67,25 & 2,90 \\
Serralha & 59,01 & 3,21 & 58,30 & 2,87 & 30,04 & 0,04 & 56,12 & 2,22 \\
Taioba & 55,54 & 1,76 & 61,05 & 2,10 & 20,06 & 0,13 & 57,68 & 1,49 \\
Vagem & 65,79 & 0,27 & 59,21 & 0,20 & 25,56 & 0,04 & 56,33 & 0,11 \\
\hline
\end{tabular}


Em termos de luteína, a rúcula destacou-se como a fonte mais promissora quando extraída em acetona (5,12 mg.100 g g $)$ e o lobrobô quando extraído com etanol e tetraidrofurano $\left(4,76 \mathrm{mg} \cdot 100 \mathrm{~g}^{-1} \mathrm{e}\right.$ 3,50 mg.100 g-1 , respectivamente).

A proporção de luteína em relação ao total de carotenóides é elevada nas hortaliças amargas, como, almeirão, azedinho e rúcula (Tabela 2 ).

É interessante notar que os vegetais lobrobô, serralha e taioba (hortaliças não-convencionais e de consumo regional) destacaram-se como fontes promissoras de luteína, superando até mesmo o espinafre. Outros vegetais regionais como o quiabo e o jiló não podem ser considerados como fontes desse pigmento, pois apresentaram os piores resultados com todos os solventes empregados. O mesmo pode-se afirmar para a vagem, o repolho e para os três pimentões estudados.

O agrião, a acelga, o almeirão, o azedinho, o brócolis, as couves comum e roxa, o espinafre e a mostarda, todos com teor de luteína superior a $1 \mathrm{mg} .100 \mathrm{~g} \mathrm{~g}^{-1}$ de produto, constituem boa fonte de luteína, porém são dependentes do solvente extrator.

Na maioria das hortaliças avaliadas a luteína foi o carotenóide majoritário, exceto para acelga e espinafre quando extraídas em etanol e para o pimentão amarelo nos quatro solventes extratores.

O fato da luteína não representar o carotenóide majoritário em pimentões amarelos contraria os resultados de BIANCHINI e PENTEADO (1998), que verificaram para cultivares de pimentão amarelo Zarco Híbrido $F_{1}$ e Sunboy Híbrido $F_{1}$ teores de 0,55 e 0,41 mg.100 g ${ }^{-1}$. No entanto, condiz com as afirmações de DAVIES, MATTHEWS e KIRK (1970) e MATUS, DELI e SZABOLCS (1991). Esses autores destacaram a violaxantina como o carotenóide mais abundante na referida fonte vegetal, representando 31,30 e $34,07 \%$ do total, respectivamente.

A área do pico do carotenóide majoritário presente no pimentão amarelo neste trabalho correspondeu a 48,56\% do total de carotenóides extraídos em acetona, 34,27\% em etanol, 33,44\% em hexano e $78,95 \%$ em tetraidrofurano. Como não era objetivo do trabalho e por questões de custos esse carotenóide não foi identificado, sendo possivelmente violaxantina de acordo com DAVIES, MATTHEWS e KIRK (1970) e MATUS, DELI e SZABOLCS (1991).

$\mathrm{Na}$ Tabela 3 são apresentadas as faixas de variação entre os valores verificados na literatura e os teores de luteína determinados nas hortaliças estudadas. Não foram encontradas citações na literatura a respeito da concentração de luteína nas hortaliças jiló, lobrobô e quiabo.

COSTA et al. (2003) obtiveram teor de luteína em folhas de acelga de 2,70 mg.100 g-1, concordando com a concentração determinada no presente trabalho quando se empregou acetona como solvente extrator $\left(2,68 \mathrm{mg} \cdot 100 \mathrm{~g} \mathrm{~g}^{-1}\right)$.

NIIZU e RODRIGUEZ-AMAYA (2005), KIMURA e RODRIGUEZ-AMAYA (2003) e HART e SCOTT (1995) encontraram teores de luteína em agrião de 5,60; 7,50 e 10,70 mg.100 g ${ }^{-1}$, respectivamente. Os valores inferiores obtidos neste trabalho $\left(3,17 ; 2,41 ; 0,31\right.$ e 2,95 mg.100 g $\left.{ }^{-1}\right)$ podem ser justificados pela maior quantidade de caule presente nas amostras analisadas ou variações decorrente das espécies avaliadas.

O teor de luteína no almeirão extraído com etanol (2,98 mg.100 g-1) e acetona (2,69 mg.100 g $\left.{ }^{-1}\right)$ mostrou-se superior ao encontrado por RODRIGUEZ-AMAYA (1993). No entanto, ao ser extraído com tetraidrofurano $\left(0,76 \mathrm{mg} .100 \mathrm{~g}^{-1}\right)$ a concentração do pigmento enquadrou-se na faixa de 0,70 a 1,00 mg.100 g-1 citada pela referida autora. Já a extração com hexano ficou muito aquém desses valores $\left(0,24 \mathrm{mg} \cdot 100 \mathrm{~g}^{-1}\right)$.

Ao avaliar o conteúdo de luteína presente em azedinho VARDAVAS et al. (2006) verificaram teor de 2,45 mg.100 g-1, muito acima das concentrações obtidas no presente trabalho $(1,66 ; 0,60$; 0,01 e $2,01 \mathrm{mg} \cdot 100 \mathrm{~g}^{-1}$ ).

Os teores de luteína nas amostras de brócolis, extraídas com etanol estão de acordo com a concentração de 1,61 mg.100 g g $^{-1}$ citada por HART e SCOTT (1995) e FRASER e BRAMLEY (2004). Também coincide com o valor de 1,80 mg.100 g ${ }^{-1}$ reportado por PRATT (1999). Porém, são inferiores aos valores de 2,40 e 2,83 mg.100 g ${ }^{-1}$ encontrados por KHACHIK, BEECHER e SMITH (1995), SOUTHON e FAULKS (2003), ALVES-RODRIGUES e SHAO (2004) e KRINSKY e JONHSON (2005). 


\section{TABELA 3 - COMPARATIVO ENTRE OS VALORES DE LUTEÍNA (mg.100 g-1) ENCONTRADOS E DADOS FORNECIDOS PELA LITERATURA}

\begin{tabular}{|c|c|c|c|}
\hline Hortaliça & Experimento * & Teores da literatura & \multirow{11}{*}{$\begin{array}{c}\text { Referências } \\
\text { (COSTA et al., 2003) } \\
\text { (HART e SCOTT, 1995; KIMURA e } \\
\text { RODRIGUEZ-AMAYA, 2002; NIIZU e } \\
\text { RODRIGUEZ-AMAYA, 2001) } \\
\text { (RODRIGUEZ-AMAYA, 1993) } \\
\text { (VARDAVAS et al., 2006) } \\
\text { (HART e SCOTT, 1995; KHACHIK, } \\
\text { BEECHER e SMITH, 1995; PRATT, } \\
\text { 1999; SOUTHON e FAULKS, 2003; } \\
\text { ALVES-RODRIGUES e SHAO, 2004; } \\
\text { FRASER e BRAMLEY, 2004; } \\
\text { KRINSKY e JONHSON, 2005) } \\
\text { (MERCADANTE e RODRIGUEZ- } \\
\text { AMAYA, 1991; RODRIGUEZ-AMAYA, } \\
\text { 1993; ALVES-RODRIGUES e SHAO, } \\
\text { 2004) } \\
\text { (MERCADANTE e RODRIGUEZ- } \\
\text { AMAYA, 1991; RODRIGUEZ-AMAYA, } \\
\text { 1993; ALVES-RODRIGUES e SHAO, } \\
\text { 2004) } \\
\text { (KHACHIK, BEECHER e SMITH, } \\
\text { 1995; KONINGS e ROOMANS, 1997; } \\
\text { SOUTHON e FAULKS, 2003; ALVES- } \\
\text { RODRIGUES e SHAO, 2004; } \\
\text { FRASER e BRAMLEY, 2004; } \\
\text { KRINSKY e JHONSON, 2005; } \\
\text { VARDAVAS et al., 2005) }\end{array}$} \\
\hline Acelga & $1,48-2,63$ & 2,70 & \\
\hline Agrião & $2,41-3,17$ & $5,60-10,70$ & \\
\hline Almeirão & $0,76-2,98$ & $0,70-1,00$ & \\
\hline Azedinho & $0,60-2,01$ & 2,45 & \\
\hline Brócolis & $0,76-1,84$ & $1,61-2,83$ & \\
\hline $\begin{array}{l}\text { Couve } \\
\text { comum }\end{array}$ & $1,95-3,51$ & $1,30-39,50$ & \\
\hline Couve roxa & $0,41-3,64$ & $1,30-39,50$ & \\
\hline & & & \\
\hline Espinafre & $0,43-2,21$ & $3,05-11,94$ & \\
\hline & & & \\
\hline Jiló & $0,01-0,09$ & ne & \multirow[b]{3}{*}{$\begin{array}{c}\text { (KAHCHIK, BEECHER e SMITH, } \\
1995)\end{array}$} \\
\hline Lobrobô & $3,10-4,76$ & ne & \\
\hline Mostarda & $0,97-2,47$ & 7,41 & \\
\hline $\begin{array}{l}\text { Pimentão } \\
\text { amarelo }\end{array}$ & $0,06-0,29$ & $0,41-0,55$ & \multirow{4}{*}{$\begin{array}{l}\text { (BIANCHINI e PENTEADO, 1998) } \\
\text { (HART e SCOTT, 1995; NIIZU e } \\
\text { RODRIGUEZ-AMAYA, 2005) } \\
\text { (SOUTHON e FAULKS, 2003) }\end{array}$} \\
\hline $\begin{array}{l}\text { Pimentão } \\
\text { verde }\end{array}$ & $0,39-0,86$ & $0,66-0,77$ & \\
\hline $\begin{array}{l}\text { Pimentão } \\
\text { vermelho }\end{array}$ & $0,39-0,76$ & 0,075 & \\
\hline Quiabo & $0,26-0,73$ & ne & \\
\hline Repolho & $0,01-0,03$ & $0,15-0,31$ & \multirow{5}{*}{$\begin{array}{c}\text { (KHACHIK, BEECHER e SMITH, } \\
\text { 1995; PRATT, 1999; KRINSKY e } \\
\text { JHONSON, 2005) } \\
\text { (TASSI, 2001; KIMURA e } \\
\text { RODRIGUEZ-AMAYA, 2003; NIIZU e } \\
\text { RODRIGUEZ-AMAYA, 2005; } \\
\text { VARDAVAS et al., 2006) } \\
\text { (MERCADANTE e RODRIGUEZ- } \\
\text { AMAYA, 1991) } \\
\text { (MERCADANTE e RODRIGUEZ- } \\
\text { AMAYA, 1991) } \\
\text { (HART e SCOTT, 1995; KHACHIK, } \\
\text { BEECHER e SMITH, 1995; ALVES- } \\
\text { RODRIGUES e SHAO, 2004; } \\
\text { FRASER e BRAMLEY, 2004) }\end{array}$} \\
\hline Rúcula & $1,96-5,12$ & $0,80-5,22$ & \\
\hline Serralha & $2,22-3,21$ & $14,50^{* *}$ & \\
\hline Taioba & $1,49-2,10$ & $17,20^{\star *}$ & \\
\hline Vagem & $0,11-0,27$ & $0,47-0,64$ & \\
\hline
\end{tabular}

\footnotetext{
* Os valores para as extrações com hexano não foram levados em consideração ao construir a tabela, pois o teor de luteína das hortaliças poderia ser subestimado.

** Teores expressos em termos de luteína + violaxantina.

ne $=$ não-encontrado.
} 
Verificou-se grande discrepância nos dados da literatura com relação aos teores de luteína presentes em couves, incluindo variação entre 1,30 a 2,00 mg.100 g-1 (RODRIGUEZ-AMAYA, 1993)

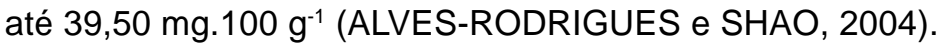

MERCADANTE e RODRIGUEZ-AMAYA (1991) verificaram que até a estação do ano influencia significativamente os teores de carotenóides em couve. No verão, os valores são menores que no inverno em função de temperaturas elevadas e maior intensidade luminosa. Constatou-se que a de luteína na couve roxa (68,60; 83,02 e 62,90\%) é superior à proporção apresentada pela couve comum $(44,40 ; 56,15$ e $61,66 \%)$ quando extraída com acetona, etanol e tetraidrofurano.

O valor encontrado para o espinafre variou de 0,43 a 2,21 mg.100 $\mathrm{g}^{-1}$, dependendo do solvente, e diferiu dos teores citados na literatura (também discrepantes entre si). Segundo VARDAVAS et al. (2006), o espinafre contém 3,05 mg de luteína.100 $\mathrm{g}^{-1}$. Já KONINGS e ROOMANS (1997), KHACHIK, BEECHER e SMITH, (1995), FRASER e BRAMLEY (2004) e SOUTHON e FAULKS (2003) concordam com valores superiores variando entre 5,0 e 6,2 mg.100 g $\mathrm{g}^{-1}$. KRINSKY e JHONSON (2005) e ALVES-RODRIGUES e SHAO (2004) encontraram 11,94 mg de luteína.100 g-1 para o espinafre.

KHACHIK, BEECHER e SMITH (1995) constataram teor de luteína de 7,41 mg.100 g-1 na mostarda, muito superior ao verificado nesse trabalho $\left(0,04\right.$ a 2,47 mg.100 $\left.\mathrm{g}^{-1}\right)$.

HART e SCOTT (1995) e NIIZU e RODRIGUEZ-AMAYA (2005) consideraram o pimentão verde como fonte pobre em carotenóides. A concentração de luteína encontrada no pimentão verde extraído com etanol $\left(0,86 \mathrm{mg} .100 \mathrm{~g}^{-1}\right)$ superou a citada pelos autores $\left(0,66\right.$ e $\left.0,77 \mathrm{mg} \cdot 100 \mathrm{~g} \mathrm{~g}^{-1}\right)$. No entanto, o teor foi inferior ao obtido em estudos anteriores em extrações com acetona, hexano e tetraidrofurano $\left(0,39 ; 0,14\right.$ e $\left.0,43 \mathrm{mg} \cdot 100 \mathrm{~g}^{-1}\right)$.

SOUTHON e FAULKS (2003) obtiveram concentração de luteína de $0,075 \mathrm{mg} .100 \mathrm{~g}^{-1} \mathrm{em}$ pimentões vermelhos, valor aquém dos encontrados para as extrações com acetona, etanol e tetraidrofurano $\left(0,41 ; 0,75\right.$ e $\left.0,39 \mathrm{mg} \cdot 100 \mathrm{~g}^{-1}\right)$, mas próximo ao verificado na extração em hexano $\left(0,07 \mathrm{mg} .100 \mathrm{~g}^{-1}\right)$.

Para HART e SCOTT (1995), o conteúdo de carotenóides em fontes vegetais depende da quantidade e do tipo de material utilizado no preparo da amostra. Afirmaram que dependendo da folha de repolho analisada pode ocorrer variação de até 150 vezes no teor obtido. Comparando os dados da Tabela $1 \mathrm{com}$ a literatura observou-se que o teor de luteína do repolho extraído com

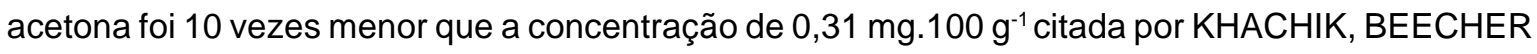
e SMITH (1995) e KRINSKY e JHONSON (2005). Já PRATT (1999) encontrou valor 15 vezes superior para repolho com os solventes etanol, hexano e tetraidrofurano $\left(0,010 \mathrm{mg} .100 \mathrm{~g}^{-1}\right)$. As variações entre o presente estudo e o desenvolvido por PRATT (1999) podem ser justificadas pelo uso de folhas internas, nas quais a concentração de luteína é menor que nas externas.

Nenhuma das extrações realizadas na vagem excedeu o valor de $0,49 \mathrm{mg}^{100} \mathrm{~g} \mathrm{~g}^{-1}$ encontrado por FRASER e BRAMLEY (2004), a concentração de 0,59 mg.100 g $\mathrm{g}^{-1}$ determinada por KHACHIK, BEECHER e SMITH (1995), a faixa de 0,47-0,55 mg.100 g-1 citada por HART e SCOTT (1995) e o teor de 0,64 mg.100 g-1 obtido por ALVES-RODRIGUES e SHAO (2004).

O teor de luteína na rúcula extraída com acetona (5,12 mg.100 g $\left.\mathrm{g}^{-1}\right)$ está de acordo com os valores de 5,00; 5,30 e 5,22 mg.100 g-1 encontrados por NIIZU e RODRIGUEZ-AMAYA (2005), TASSI (2001) e KIMURA e RODRIGUEZ-AMAYA (2003). Já as extrações com etanol e tetraidrofurano (1,96 mg.100 g-1 e 2,90 mg.100 g g $)$ coincidiram com a de VARDAVAS et al. (2006) (2,48 mg.100 g $\left.{ }^{-1}\right)$.

MERCADANTE e RODRIGUEZ-AMAYA (1991) determinaram a concentração de carotenóides presentes em serralha $\left(14,50 \mathrm{mg} .100 \mathrm{~g}^{-1}\right)$ e taioba $\left(17,20 \mathrm{mg} .100 \mathrm{~g}^{-1}\right)$. Porém com valores expressos em termos de luteína + violaxantina impossibilitando a comparação.

A variação entre os valores obtidos neste trabalho e os citados na literatura pode ser justificada pela parte da planta analisada, estágio de maturação, condições edafoclimáticas, manejo pós-colheita, variedade e espécie analisada. De qualquer forma, saladas cruas dessas hortaliças fornecem quantidades elevadas de luteína com exceção do quiabo, pimentão, vagem, jiló e repolho. 
A dificuldade para comparar os resultados obtidos com a literatura decorre de ampla faixa de valores citados e diferentes unidades utilizadas para expressar o teor de luteína ( $\mu \mathrm{g} .100 \mathrm{~g}^{-1}, \mu \mathrm{g} \cdot \mathrm{g}^{-1} \mathrm{e}$ mg.100 g ${ }^{-1}$, incluindo dados da literatura (FRASER e BRAMLEY, 2004) transformados de forma errônea.

\section{CONCLUSÃO}

A escolha do solvente extrator exerce papel fundamental na análise de carotenóides em alimentos.

Os dados obtidos permitem concluir que acelga, agrião, azedinho, brócolis, couve, espinafre, mostarda e rúcula são vegetais ricos em luteína. Portanto, seu consumo pode contribuir significativamente para a defesa do organismo contra doenças degenerativas.

Encontrou-se elevado teor de luteína no almeirão, lobrobô, serralha e na taioba, vegetais nãoconvencionais consumidos pela população interiorana (notadamente em Minas Gerais). Esse fato merece divulgação para ampliar o consumo das referidas fontes vegetais, visando propagação da saúde.

\section{ABSTRACT}

\section{DETERMINATION OF LUTEIN CONTENT IN VEGETABLES}

The objective of the current research was to evaluate the concentration of lutein in vegetables commonly consumed in the State of Minas Gerais (BRAZIL), aiming to provide data so the population can know which food presents the highest sources of this carotenoid. The extraction of the pigment was carried out in acetone, ethanol, hexane and tetrahydrofurane solutions for 24 hours. After the extraction, a saponification in $10 \%$ ethanolic $\mathrm{KOH}$ occurred to remove impurities such as clorophyl and lipidic acids. The quantity of lutein in the vegetables was determined by spectrophotometric measures, later confirmed by High Performance Liquid Chromatography (HPLC). From the results obtained, it was observed that acetone was the most effective solvent to extract the analysed pigment while the hexane solution was not effective to extract it from fresh vegetables at this conditions. Vegetables such as cabbage, rucola, mustard, chard, spinach, azedinho and broccoli constitute a rich source of Lutein. It is important to emphasize the high concentration of this carotenoid found in lobrobô, also known as ora-pro-nobis, as well as in serralha, chicory and taioba. However, those former mentioned vegetables are consumed only at some specific Brazilian regions.

KEY-WORDS: LUTEIN; CAROTENOIDS; VEGETABLES.

\section{REFERÊNCIAS}

1 ALVES-RODRIGUES, A.; SHAO, A. The science behind lutein. Toxicology Letters, n. 150, p. 57-83, 2004.

2 BIANCHINI, R.; PENTEADO, M. de V. C. Carotenóides de pimentões amarelos (Capsicum annuum) caracterização e verificação de mudanças com o cozimento. Ciência e Tecnologia de Alimentos, Campinas, v. 18, n. 3, p. 283-288, ago/ out. 1998.

3 BROW, L.; RIMM, E. B.; SEDDON, J. M.; GIOVANUCCI, E. L.; CHASAN-TABER, L.; SPIEGELMAN, D.; WILLET, W. C.; HANKINSON, S. E. A prospective study of carotenoid intake and risk of cataract extraction in US men. American Journal Clinical of Nutritional, n. 70, p. 517-524, 1999.

4 CHASAN-TABER, L.; WILLET, W. C.; SEDDON, J. M.; STAMPFER, M. J.; ROSNER, B.; COLDITZ, G. A.; SPEIZER, F. E.; HANKINSON, S. E. A prospective study of carotenoid and vitamin A intakes and risk of cataract extraction in US women. American Journal Clinical of Nutritional, n. 70, p. 509-516, 1999.

5 CISNEROS, M.; BENAVIDES, J.; BRENES, C. H.; RITO-PALOMARES, M. Recovery in aqueous two-phase systems of lutein produced by the green microalga Chlorella protothecoide. Journal of Chromatography B, v. 807, n.1, p. 105-110, 2004.

6 COSTA, S. M. de; MONTENEGRO, M. A.; ARREgUI, T.; PINTO, M. I. S. de; NAZARENO, M. A.; MISHIMA, B. L. de. Characterization of fresh Beta vulgaris from Santiago del Estero (Argentina). Nutrient and caroteniod content of stem and leaves. Ciência \& Tecnologia de Alimentos, Campinas, v. 23, n.1, p. 33-37, jan./abr.2003. 
7 CRAFT, N. E. Relative solubility, stability, and absorptivity of lutein and $\beta$-carotene in organic solvents. Journal Agriculture of Food Chemistry, v. 40, p. 431-434, 1992.

8 DAGNELIE, G.; ZORGE, I.; McDONALD, T. M. Lutein improves visual function in some patients with retinal degeneration: a pilot study via the internet. Optometry, n. 71, p. 147-164, 2000.

9 DAVIES, B. H.; MATTHEWS, S.; KIRK, J. T. O. The nature and biosynthesis of the carotenoids of different colour cultivars of Capsicum annuum. Phytochemistry, v.9, p.797-805, 1970.

10 DELI, J.; MOLNÁR, P.; OSZ, E.; TÓTH, G.; ZSILA, F. Epimerisation of lutein to 3'-epilutein in processed foods. Bioorganic \& Medicinal Chemistry Letters, n.14, p. 925-928, 2004.

11 FRASER, P. D.; BRAMLEY, P. M. The biosynthesis and nutritional uses of carotenoids. Progress in Lipid Research, n. 43, p. 228-265, 2004.

12 HART, D. J.; SCOTT, K. J. Development and evaluation of an HPLC method for the analysis of carotenoids in foods, and the measurement of the carotenoid content of vegetables and fruits commonly consumed in the UK. Food Chemistry, v. 54, p. 101-111, 1995.

$13 \mathrm{KHACHIK}, \mathrm{F}$; BEECHER, G. R.; SMITH, C. Jr. Lutein, lycopene, and their oxidative metabolites in chemoprevention of cancer. Journal of Cellular Biochemistry, n. 22, p. 236-246, 1995.

14 KIMURA, M.; RODRIGUEZ-AMAYA D. B. A scheme for obtaining standards and HPLC quantification of leafy vegetable carotenoids. Food Chemistry, n. 78, p. 389-398, 2002.

15 KONINGS, E. J. M.; ROOMANS, H. H. S. Evaluation and validation of an LC method for the analysis of carotenoids in vegetables and fruit. Food Chemistry, v. 59, n. 4, p. 599-603, 1997.

16 KRINSKY, N. I.; JONHSON, E. J. Carotenoid actions and their relation to health and disease. Molecular Aspects of Medicine, v. 26, p. 459-516, 2005.

17 LAJOLO, F. Zeaxantina e luteína reduzem riscos de degeneração macular. Disponível em: <http:// nutricaoempauta.locaweb.com.br/lista_artigo.php?cod=325>. Acesso em: 01 jun. 2004.

18 MATUS, Z.; DELI, J.; SZABOLCS, J. Carotenoid composition of yellow pepper during ripening: isolation of beta-criptoxantin 5,6-epoxide. Journal of Agriculture Food Chemistry, v.39, p.1907-14, 1991.

19 MERCADANTE, A. Z.; RODRIGUEZ-AMAYA, D. B. Carotenoid composition and vitamin A value of some native Brazilian green leafy vegetables. Journal Food Science Technology, v. 25, p.213-219, 1991.

20 MERCADANTE, A. Z.; RODRIGUEZ-AMAYA, D. B. Confirmação da identidade da $\alpha$-criptoxantina e incidência de carotenóides minoritários provitamínicos A em verduras folhosas verdes. Ciência e Tecnologia de Alimentos, Campinas, v.21, n.2, p. 216-222, mai./ago. 2001.

21 NIIZU, P. Y.; RODRIGUEZ-AMAYA, D. B. New data on the carotenoid composition of raw salad vegetables. Journal of Food Composition and Analysis, v.18, p. 739-749, 2005.

22 NUNES, I. L.; MERCADANTE, A. Z: Utilização de colunas de fase reversa $C_{18}$ e $C_{30}$ para separação de carotenóides por CLAE. In: CONGRESSO BRASILEIRO DE CIÊNCIA E TECNOLOGIA DE ALIMENTOS, 19., 2004, Recife/PE. Anais... Recife: SBCTA/UFPE, 2004. CD-Rom.

23 PEREIRA, A. S. Teores de carotenóides em cenoura (Daucus carota L.) e sua relação com a coloração das raízes. Viçosa, 2002. 128 f. Tese (Doutorado em Ciência e Tecnologia de Alimentos) - Centro de Ciências Exatas, Universidade Federal de Viçosa.

24 PRATT, S. M. D. Dietary prevention of age-related macular degeneration. Journal of the American Optometric Association, v. 70, p.39-47, 1999.

25 RODRIGUEZ-AMAYA, D. B. Nature and distribution of carotenoids in foods. In: CHARALAMBOUS, F. Shelf life studies of foods and beverages- chemical, biological, physical and nutritional aspects. Amsterdam: Elsevier Science, 1993. p. 547-589.

26 RODRIGUEZ-AMAYA, D. B. A Guide to carotenoid analysis in foods. Washington: ILSI, 2001.64 p.

27 SÁ, M. C. de; RODRIGUEZ-AMAYA, D. B. Carotenoid composition of cooked green vegetables from restaurants. Food Chemistry, v. 83, p. 595-600, 2003.

28 SILVA, A. S. Luteína, alimento para tu vista. Food Ingredients, v. 6, p. 80-81, 2004

29 STRUNCH, S. S. La luteína: el antioxidante para los ojos que envejecen. Disponível em: <http://retinosis.org/ articulo.php?sec=medicina\&doc=luteina.htm>. Acesso: 19 maio 2004.

30 SOUTHON, S.: FAULKS, R. Carotenoids in food: bioavailability and functional benefits. In: Phytochemical functional foods. Chicago: Woodhead Publishing \& CRC Press LLC, 2003. chap. 7. 
31 SUMANTRAN, V. N.; ZHANG, R.; LEE, D. S.; WICHA, M. S. Differential regulation of apoptosis in normal versus transformed mammary epithelium by lutein and retinoic acid. Cancer Epidemiology, Biomarkers \& Prevention, v. 9, p. 257-263, 2000.

32 TASSI, E. M. M. Caracterização química, atividade da lipoxigenase e biodisponibilidade de carotenóides da rúcula (Eruca sativa). Campinas 2001. 107 f. Tese (Doutorado em Alimentos e Nutrição) - Engenharia de Alimentos, Universidade Estadual de Campinas.

33 VARDAVAS, C. I.; MAJCHRZAK, D.; WAGNER, K. H.; ELMADFA, I.; KAFATOS, A. The antioxidant and phylloquinone content of wildly grown greens in Crete. Food Chemistry, v. 99, n. 4, p. 813-821, 2006.

34 YEUM, K.; TAYLOR, A.; TANG, G.; RUSSELL, R. M. Measurement of carotenoids, retinoids, and tocopherol in human lenses. Investigative Ophthalmology \& Visual Science, v. 36, n. 13, p. 2756-2761,1995.

35 ZONTA, E. P.; MACHADO, A. A. Manual do SANEST: sistema de análise estatística para microcomputadores. Pelotas: UFPel, 1991. $102 \mathrm{p}$. 